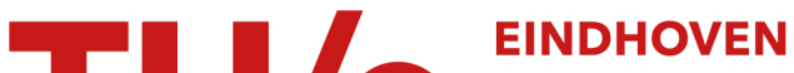 UNIVERSITY OF TECHNOLOGY
}

\section{Combined position \& force control of a robotic manipulator}

\section{Citation for published version (APA):}

Sijs, J., Liefhebber, F., \& Römer, G. W. R. B. E. (2007). Combined position \& force control of a robotic manipulator. In Proceeding of the 10th IEEE International Conference on Rehabilitation Robotics (ICORR '07), June 13-15 2012, Noordwijk, The Netherlands (pp. 106-111). Institute of Electrical and Electronics Engineers. https://doi.org/10.1109/ICORR.2007.4428414

DOI:

10.1109/ICORR.2007.4428414

Document status and date:

Published: 01/01/2007

\section{Document Version:}

Publisher's PDF, also known as Version of Record (includes final page, issue and volume numbers)

\section{Please check the document version of this publication:}

- A submitted manuscript is the version of the article upon submission and before peer-review. There can be important differences between the submitted version and the official published version of record. People interested in the research are advised to contact the author for the final version of the publication, or visit the $\mathrm{DOI}$ to the publisher's website.

- The final author version and the galley proof are versions of the publication after peer review.

- The final published version features the final layout of the paper including the volume, issue and page numbers.

Link to publication

\section{General rights}

Copyright and moral rights for the publications made accessible in the public portal are retained by the authors and/or other copyright owners and it is a condition of accessing publications that users recognise and abide by the legal requirements associated with these rights.

- Users may download and print one copy of any publication from the public portal for the purpose of private study or research.

- You may not further distribute the material or use it for any profit-making activity or commercial gain

- You may freely distribute the URL identifying the publication in the public portal.

If the publication is distributed under the terms of Article $25 \mathrm{fa}$ of the Dutch Copyright Act, indicated by the "Taverne" license above, please follow below link for the End User Agreement:

www.tue.nl/taverne

Take down policy

If you believe that this document breaches copyright please contact us at:

openaccess@tue.nl

providing details and we will investigate your claim. 


\title{
Combined Position \& Force Control for a robotic manipulator
}

\author{
Joris Sijs, Freek Liefhebber and Gert Willem R.B.E. Römer
}

\begin{abstract}
The ARM is a 6 DOF robotic manipulator used by disabled people with a severe handicap at the upper extremities The present ARM is position and velocity controlled. The desired position of the robot is given by the user. However, in constraint scenario's, manipulation becomes too difficult and an asistant-controller is wanted. This assistant is based on external forces on the gripper of the robot, measured using a force-torque sensor. A new control strategy is designed for measured forces and user input. The basic principle of this strategy is derived from the way that humans steer their hand. Sensed forces are followed until they are notpresent anymore, except when the user wants to do a manipulation in that direc tion. Therefore a combined positionforce controller was designed. All 6 DOF of the robot can be steered by both the user and the force controller at the same time. Beside the design of the control strategy, it is also implemented on the ARM and tested in four test-cases.
\end{abstract}

\section{INTRODUCTION}

$\mathrm{P}$ EOPLE with a physical handicap become more independent when using a robot arm such as the ARM (Assistive Robotic Manipulator), previously known as Manus [6]. It is an assistive robotic manipulator with 6 Degrees of Freedom (DOF). The gripper of the robot is operated using a variety of input-devices including a joystick and a keypad. The robot allows the user to carry out all-dayliving (ADL) tasks independently. However, a number of scenario's are too difficult to complete the task or manipulation. In such scenario's the robot must follow a constraint path in multiple directions at the same time to complete the task. Constraint scenario's like opening a bottle and opening a door demand that the ARM is steered in two or more directions. This paper discusses a control approach to cope with these scenario's using an automatic force controller. To that end a commercial force sensor [1], was mounted between the wrist and the gripper of the ARM. Its position is shown in Figure 1

In previous work, a hybrid position/force controller was designed $[2,5]$. This controller allowed the user to choose which DOF was controlled by the position controller and which (other DOF) was controlled by the force controller. This control approach proved to be too difficult for the user,

Manuscript received Jaruary 31, 2007.

$\mathrm{J}$. Sijs is with TNO Science and Industry, $2600 \mathrm{AD}$ Delft, Netherlands (corresponding author to provide phore: +31 (0)15-2692217; fax: +31 (0)15-2692111; e-mail: joris.sijs(d)tno.nl).

F. Liefhebber is with TNO Science and Inchstry, $2600 \mathrm{AD}$ Delft, Netherlands (corresponding author to provide phone: +31 (0)15-2692267; fax: +31 (0)15-2692111; e-mail: freek.liefhebber(0) tro.nl)

G.RB E. Römer is with Exact Dymarnics, 6814 CS Amhem, Netherlands (corresponding author to provide phone: +31 (0)316-334114; fax: +31 (0)316 331327; e-mail: re search(d)exactdynamics.rl).

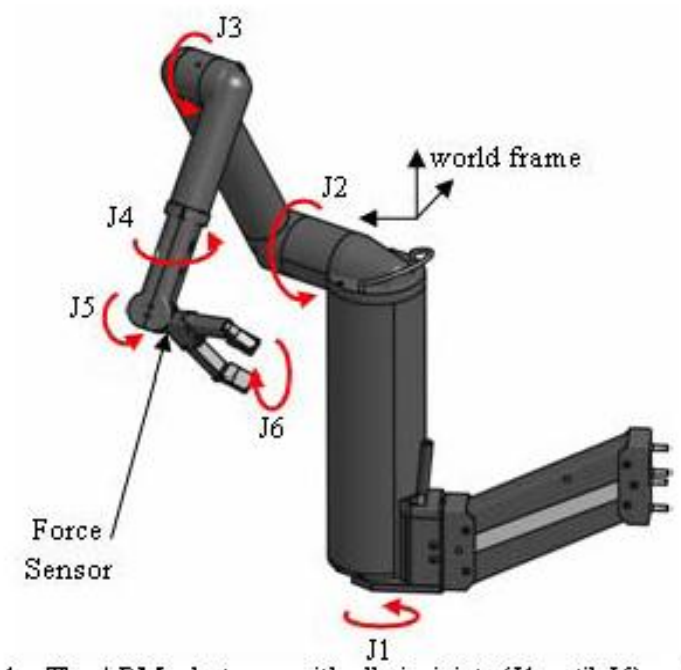

Fig. 1. The ARM robot arm with all six joints (J1 until J6) and the position of the force sensor.

because it depends on the task whether a DOF is best controlled in position or in force mode. Therefore another controller was designed in which all DOF's are controlled by both the position (user) as well as the force controller (force sensor). This paper presents the design, implementation and testing of a simultaneous position/force controller used for a robotic manipulator. In section II the system setup is discussed. The third paragraph describes the designed control strategy. This strategy is implemented on the ARM using Matlab Simulink and tested in four cases which are described in the fourth section.

\section{SYSTEM DESCRIPTION}

\section{A. ARM with position controller}

The position controller of the ARM calculates a desired position (set-point) $x_{\text {des }}$ expressed in a Cartesian coor dinates of the world frame $x_{d e s}=(x, y, z$, roll, pitch, yow $)$. The base of the world frame is at the shoulder of the ARM (position of $\mathrm{J} 2$ in Figure 1).

The user steers the ARM by giving speed in a certain DOF $v_{\text {wer }}$ which is integrated by the position controller, yielding $x_{\text {des }}$ The maximum speed that the user can give in any direction is $0.1 \mathrm{~m} / \mathrm{s}$ for translation in the $\mathrm{x}-, \mathrm{y}$ - and $\mathrm{z}$ direction and $1 \mathrm{rad} / \mathrm{s}$ for rotation around the $\mathrm{x}-, \mathrm{y}$ - and $\mathrm{z}$-axis [2].

\section{B. ARM with simultaneous position/force controller}

The control of the ARM by the user is eased by using the information of external forces acting on the gripper. To that end a force sensor was mounted between the wrist and the 
gripper of the robot. The sensor is a Mini45 SI-580-20 from ATI of Apex, USA [1]. This sensor is able to measure forces as well as torques in all $6 \mathrm{DOF}$. The measured forces are transformed into Cartesian coordinates of the world frame $F_{\text {sen }}$, see figure 2 .

The signal from the user $v_{\text {user }}$ is used as input for a position controller, which calculates a desired position according to the user $x_{\text {wser }}$. The signal from the force sensor $F_{\text {sen }}$ is used as input for the force-controller. This controller also calculates a desired position $x_{\text {force }}$ only then according to external forces acting on the gripper. The desired position from both the position and force controller are added together to give the final desired position in Cartesian coordinates $x_{\text {des }}$, also referred to as admittance control or external force control [7]. A block diagram of the total system is shown in Figure 2.

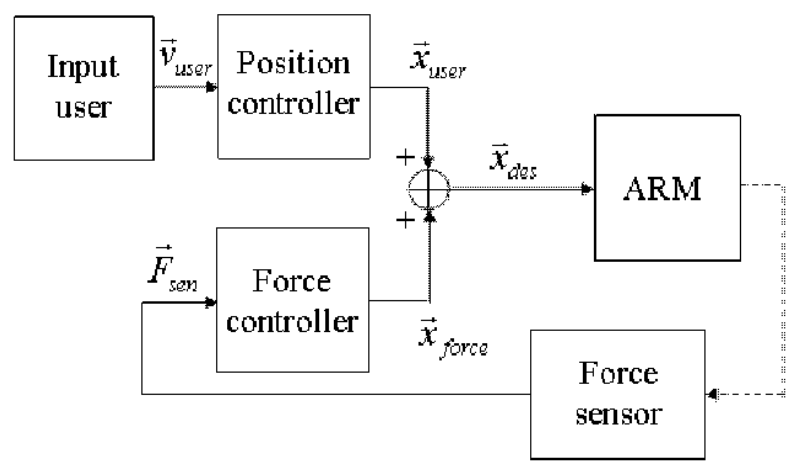

Fig. 2. Block diagram of the combined position/force controller and the ARM

\section{FORCE CONTROL}

\section{A. Problem description}

Common strategies for force control are based on Newton's law $F=m a$ [2-4] The basic principle of these hybrid position/force controllers are dividing the controllable DOF into a part that is controlled by the position controller and a part that is controlled by the force controller. The disadvantage of such a control strategy is that for every task the user has to decide and assign which DOF should be controlled by the position controller and which by the force controller.

Humans have a different control strategy. A person always follows (or gives way to) the external forces acting on his or her hand, except when the person wants to make a manipulation in a certain direction or apply a force. An example is opening a door; when a human opens a door he or she will pull the door towards him or her (see figure 3). The person does not care that their hand will also go sideways because of the door's movement, as long as their hand is going in the direction of pulling the door..
Fig. 3. Forces when opening a door

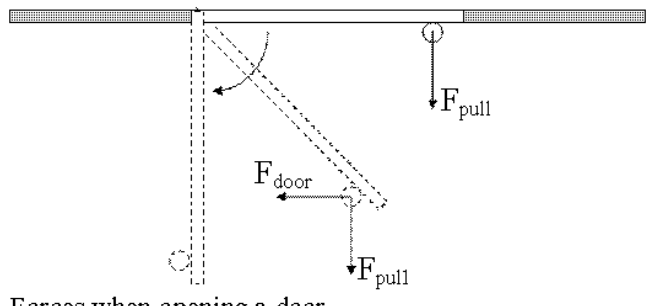

The force and direction of $F_{\text {pull }}$ is caused by the hand of the person that opens the door. However, the door will rotate and thereby creating an external force $F_{\text {door }}$ on the hand. The hand will follow the direction of the sensed force resulting in a movement of the hand to the left. If this scenario was done by a robot arm with a position controller, the user had to steer the gripper in two directions corresponding to $F_{\text {pull }}$ and $F_{\text {door }}$. This is because the robot cannot sense the force to the left and therefore cannot follow it.

\section{B. Control Strategy}

A human is able to follow the direction of forces acting on their hand. Therefore the first step in the control strategy for the ARM is to follow all external forces on the gripper. The force sensor measures all forces and their directions in Cartesian coordinates. It is tempting, as dictated by Netwon, to integrate the measured force twice and divide it by some mass to obtain a corresponding position. However, from control perspective it is better not to integrate the force on the gripper twice but only once. There are two reasons why one should integrate only once;

1. With every integration the instability of the closed control loop may grow.

2. If an external force that was acting on the gripper becomes zero instantly, it is preferable that the gripper stops (immediately) moving that direction.

An ARM with force control is able to follow all forces acting on the gripper. This means that one can grab the gripper and move it without effort in any direction. When the person grabbing the gripper stops moving the gripper and releases it, the gripper will not move. It will stay in its released position due to the single integration of the force. The ARM with only force controller is shown in Figure 4.

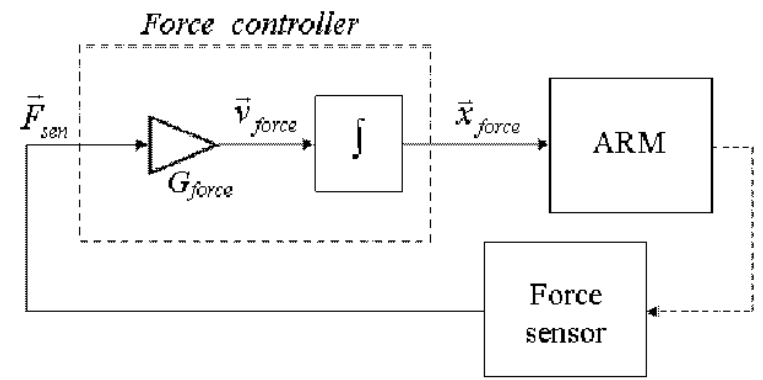

Fig. 4. Block diagram of ARM with force control

The measured forces $F_{\text {sen }}$ are multiplied with the gain $G_{\text {force }}$. The outcome of this multiplication can be considered as the desired speed according to external forces $v_{\text {force }}$. 
The second step is combining the information from the user $v_{\text {user }}$ with the information of the force sensor $F_{\text {sen }}$. The position controller will integrate $v_{\text {user }}$, yielding $x_{\text {user }}$. The desired position of to the user $x_{\text {user }}$ is added to the desired position of the force controller $x_{\text {force }}$, resulting in the final desired position $x_{\text {des. }}$. However, this is the same as adding $v_{u s e r}$ directly to $v_{\text {force }}$, shown in Figure 5 .

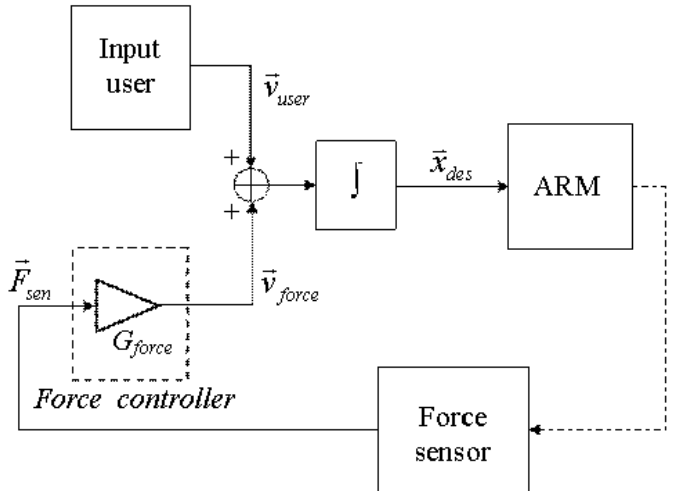

Fig. 5. Block diagram of simultaneous force/position controller

It should be noted that, for obvious reasons, the input given by the user is in any case more important then an external force on the gripper. This implies that the maximum speed commanded by the user must be bigger then the maximum speed resulting from the force controller. The maximum speed of the user is $0.1 \mathrm{~m} / \mathrm{s}$. The ARM is able to manipulate with a maximum force of $20 \mathrm{~N}$, so the signals of the force sensor $F_{\text {sen }}$ are saturated after 20N. If the gain, $G_{\text {force }}$ of Figure 5, is less then $0.1 / 20=0.05$, the speed given by the user $v_{\text {user }}$ will always be bigger then the speed given by the force controller $v_{\text {force }}$.

Three basic situations can occur;

1. The hand is moving in free space; In this case the force sensor will not measure any forces and the gripper is steered completely by the user only.

2. External force act on the gripper, which corresponds to a different direction than the direction in which the user is steering; In this situation the gripper will follow a combined direction of the user and of the external forces.

3. The gripper feels an external force which is in the same direction as the direction in which the user is steering; The speed of the user is bigger then the speed of the force controller. But because they are added together, the total speed in that specific direction will be less then the user desires. As a result the gripper slows down to a lower speed in the same direction.

Two remaining issues need to be solved before the strategy is suitable for implementation. The first issue occurs when the gripper has an object in its hand and should be stationary. In that case the force sensor will measure the object's gravity force and the gripper will follow that force while it should stand still. The second situation is when the user wants to use the robots maximum power. When the present position controlled ARM hits a wall, the error between the desired position and the real position increases rapidly. Due to this error the ARM will eventually push against the wall with its maximum force of $20 \mathrm{~N}$. When an ARM with the above position/force controller hits an the wall, the error in position will not increase rapidly due to a sensed force in the opposite direction. The result is that takes a considerable amount of time before the ARM will push with its maximum force.

The solution for these two problems is to apply a virtual box around the wrist. The gripper has an initial position inside this box. The force controller is able to position the gripper, according to the external forces, anywhere inside this box. If the gripper tents to go outside the box, the gain of the force controller $G_{\text {force }}$ is set to zero, implying that force control outside the box is not possible.

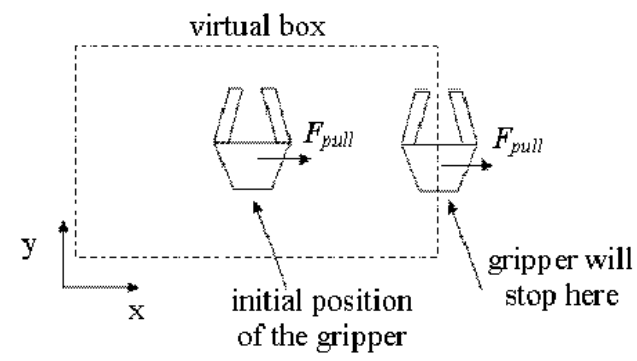

Fig. 6. Movement gripper due to force in positive $\mathrm{x}$-direction

For example, suppose the virtual box is a cubic box of size $20 \mathrm{~cm}$ and the initial position of the gripper is in the centre of the box, Figure 6 . An external force is pulling the gripper in the positive $\mathrm{x}$-direction. The gripper will follow the force until it reaches the positive plane of the virtual box at $x=10 \mathrm{~cm}$ ). No matter how big the external force, the gripper will stay on the edge of the box. Notice that the gripper will still follow external forces in the $y$ - and $z$ direction. If the external force switches to the opposite direction, the negative $\mathrm{x}$-direction, the gripper will follow this force until it reaches the other side of the box at $x=-10$ cm.

The gripper speed, commanded by the user, steers both gripper and virtual box. This means that the user can never move the wrist outside the box, because the box moves together with the gripper.

The advantages of this virtual box is explained on the basis of the two issues discussed above.

The problem with gravity was that, without the virtual box, the gripper will follow (downward) the gravity force when the robot is holding an object. When initial position of the wrist at the centre of the virtual box but already at the bottom, see Figure 7 , the gravity force is no longer able to move the wrist for this would mean that the gripper exits the box. 


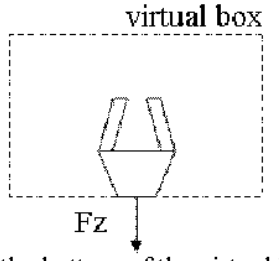

Fig. 7. Wrist situated at the bottom of the virtual box

In the second issue deals with the user wanting to apply maximum force of the robot. Without the virtual box this would take a very long time because the force controller would decrease the desired speed of the user and therefore also the force the robot applies on its environment. The following steps show the situation when the gripper (with surrounding virtual box) pushes against a solid wall, see also Figure 8 .

1. Suppose the initial position of the wrist is at the centre of the box and the gripper collides with the wall; (1) in Figure 8,

2. The user wants to push as hard as possible against the wall and will therefore steer the gripper, and also the box, even more into the wall. The box will follow the desired movement of the user into the wall, the gripper will not move into the solid wall because the wall gives an external force on the gripper in the other direction in which the gripper is pushing; (2) in Figure 8. So the gripper stands still and pushes to the wall with little force, but the virtual box is moving in the pushing direction.

3. Eventually the wrist will be at the boundary of the box and the external force due to the wall will have no influence anymore (GAIN=0). From then, only the speed of the user is acting in the direction of the wall and the robot will push with its maximum force to the wall.

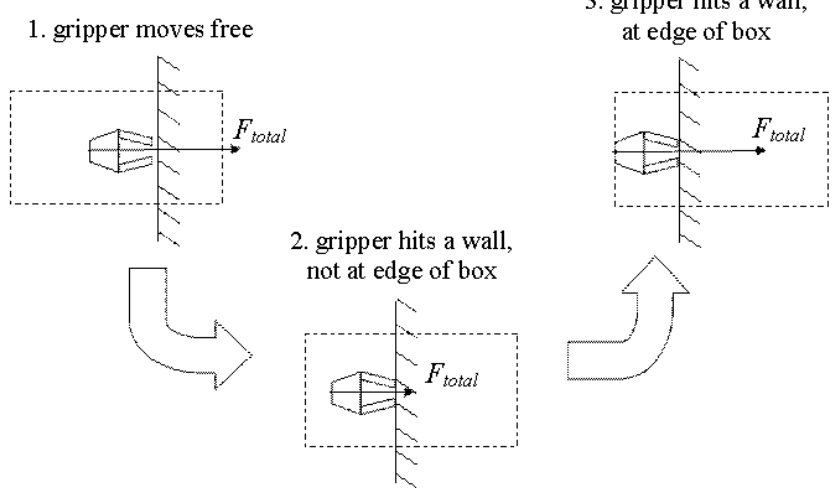

Fig. 8. Movement gripper due to gravity force; left without virtual box, right with virtual box

The best shape of the virtual box is a cubic or rectangular, because when the robot is at one plane of the box, it will still be able to follow the external forces in the direction perpendicular and parallel to this plane. This would not be the case when the box is, for example spherical. Figure 11 shows the block-scheme of this system with a simultaneous position/force controller.

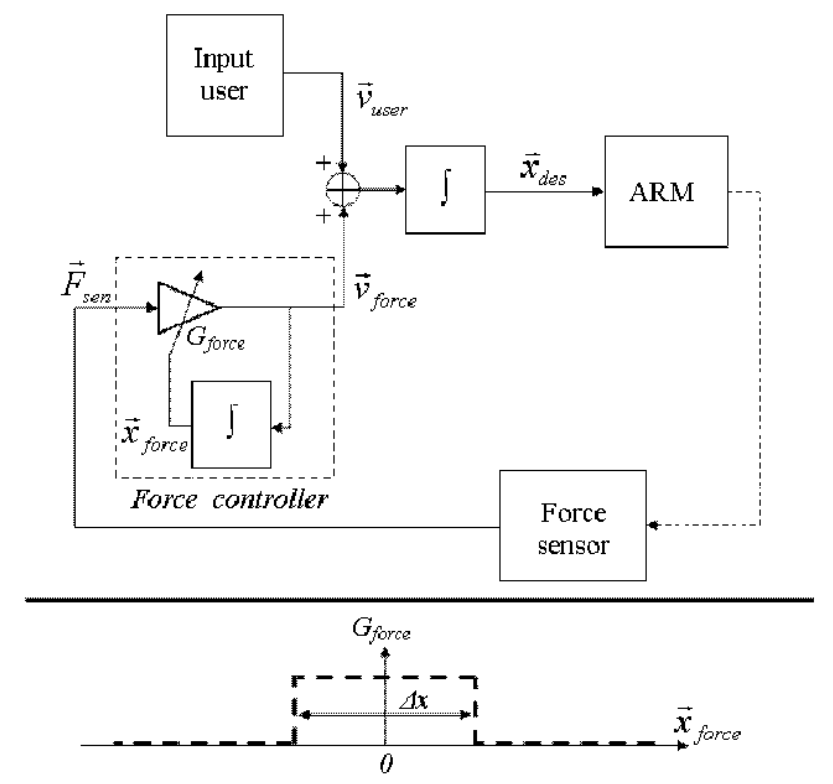

Fig. 9. Detailed block-scheme of the total system

The variable $\Delta x$ in Figure 9 represents the width of the cubic box. In the example of $G_{\text {force }}$ in Figure 9 the initial position of the wrist is at the centre of the virtual box because the centre of the rectangle is at 0 . However, the centre of this rectangle can be shifted anywhere on the axes and with that the initial position of the wrist in the virtual box..

It should be noted that this control strategy can be used in all robots, as it is a general strategy. An advantage of this strategy is that the performance of the controller does not decrease when backlash increases. Further, when the robot is burdened with friction, the robot will still follow external forces although the tracking speed will be less. Another asset of this strategy is that when an external force is removed instantly, the gripper will stay in the position it was at the moment of releasing the external force. This asset is due to the single integration of the measured force instead of a double integration. A final benefit of this strategy is that position and force act at the same time and at all times. This results in a decrease of the impact force compared to a conventional position/force controller, especially for a rigid (stiff) robotic arm. Another result is that the system is more user-friendly. The user does not have to choose which DOF is to be controlled in position and which one in force. The only thing the user has to give is whether the force controller should be on or off.

\section{TEST CASES}

The control strategy has been tested by setting up four test-cases;

- Pressing a key on a keyboard;

- Opening a door;

- Opening a turning cap;

- Placing a cup on the table. 
Each test-case will explain the benefits of the designed control strategy. In the first three test cases the strategy is tested with the forces of $F_{\text {sen }}$ only In the fourth case the strategy is tested with the torques of $F_{\text {sen }}$ only. This is done because the test-case of placing a cup on a table will show that following torques by the force controller can make a manipulation more difficult for the user instead of easier.

\section{A. Pressing a button}

Without force control the user will move the gripper towards the button, press it and pull the gripper back. However, due to friction, backlash and response-time of the user, the gripper will push the button with a certain impactforce and time. When the impact is too high it will damage the button. The same scenario holds for impact with other objects like a table. Force control can help the user by decreasing the impact-force and shorten the impact-time. In this test-case only the forces are controlled, not the torques.

In this scenario the gripper was positioned vertically above the button. The robot is steered downwards (negative $z$-axis) to press the button and upwards afterwards. This is done four times; first two times without force control, then two times with force control. Figure 10 shows the measured force on the $z$-axis.

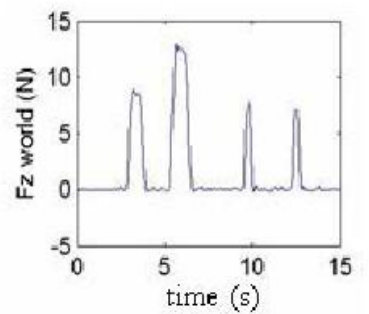

Fig. 10. Measured forces when pressing a button

The measured force of Figure 10 shows four peaks, at each peak the button was pressed by the gripper. The magnitude of impact-force is less with force control compared to the situation without force control. Also the total amount of impact-time that the gripper presses the key is less in a system with force control. The conclusion of this test-case is that the impact of a robotic manipulator with simultaneous position/force controller is decreased. and there prevents the robot from causing certain damage.

\section{B. Opening a door}

In the case of opening a door, using only a position controller, the gripper of ARM has to be steered in two directions simultaneously. With the new control strategy the aim is that the user only steers the gripper in one direction (backwards) to open the door. The gripper should follow the sideways direction with help of the force controller. Two tests were carried out, one with only position control and one with the designed position/force controller. Also in this case force control was only based on measured forces and not the measured torques of $F_{s e n}$. The results are shown in figure 11 which depicts a top-view of the position of the gripper. The ideal rotation of the door is colored grey.

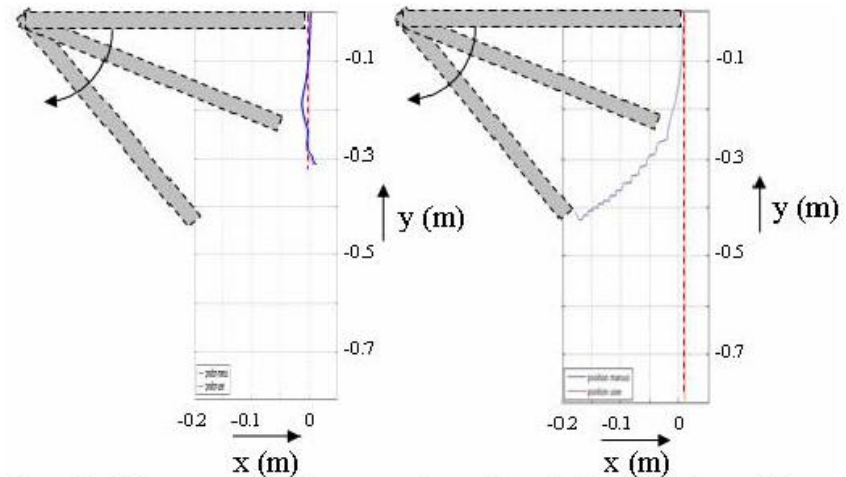

Fig. 11. Measurements when opening a door, left with only position control, right with position/force control.

The dashed line represents the integrated speed (i.e. desired position) commanded by the user. The solid line is the real (measured) position of the gripper. The left graph of Figure 11 shows the results when, only position control is active. It shows that the gripper follows the position of the user. However, the door also goes sideways. At the time that the gripper was at the end of the dashed line, the links of the robot were jerked and therefore the test was terminated. The right graph of Figure 11 shows the results of the combined position/force control. It shows that the gripper follows the movement of the door although the user only command the gripper to move in the negative y-direction. The conclusion of this test is that the designed strategy indeed helps the user to complete this difficult manipulation task.

\section{Rotating a bottle cap}

Opening a bottle by turning its bottle cap involves control of three DOF's, seen from a frame that is placed on the gripper. To open the bottle (placed vertically), the gripper must be steered around the $z$-axis of the gripper, a translation in the $\mathrm{x}$-direction and translation in the y-direction, at the same time. The same holds for opening a water tap and certain door handles. This manipulation is virtually impossible for the user to do. So force control can help. Suppose the gripper only follows measured forces and not torques. This scenario, shown in Figure 12.

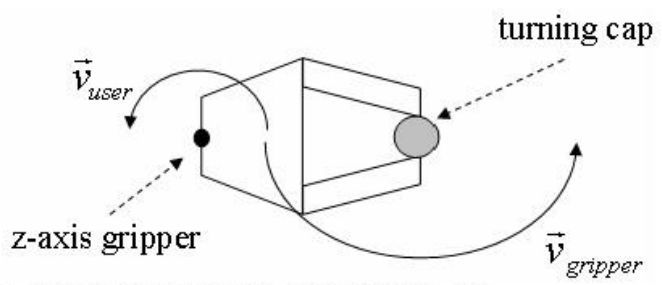

Fig. 12. Steering the gripper to open a turning cap

If the user commands the gripper to rotate about the z-axis $\left(v_{u s e r}\right)$, the sensor will measure forces in the $\mathrm{x}$ - and $\mathrm{y}$ direction. Then, the combined position/force controller steers the gripper in the correct $\mathrm{x}$ - and $\mathrm{y}$-direction to open the cap, based on the measured forces. The speed of the user and force controller combined is called $v_{\text {gripper }}$. The position of the point called ' $z$-axis gripper' in Figure 12 is measured during the test-case of opening a bottle. In an optimal situation this point will describe a circle with the turning cap 
as its centre. Figure 13 shows the result of the measurement in top-view. The dashed line is the perfect circle, the other circle represents the real position of the ' $z$-axis gripper'.

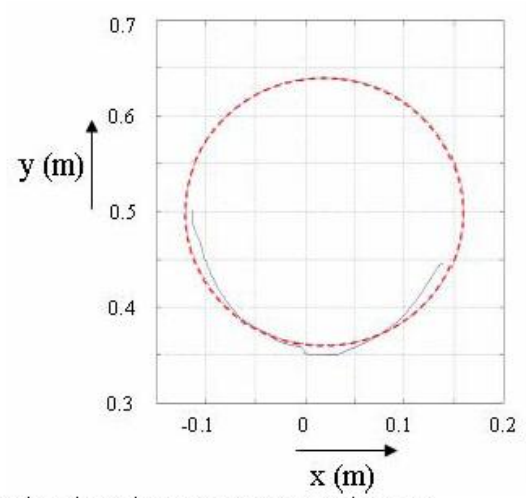

Fig. 13. Steering the gripper to open a turning cap

From Figure 13 can be concluded that in this complex manipulation, can be brought to a success with the simultaneous position/force controller.

\section{Putting a cup on the table}

In this test-case the aim is to place a cup on a table, which is not vertically clamped by the gripper, see Figure 14 . For example, the cup can be rotated during the time that the user drank from it. This should be facilitated by using the simultaneous position/force controller. Due to external torques acting on the gripper, and which are measured by the sensor, the force controller will rotate the gripper in such a way that the bottom of the cup becomes aligned with the table. In this test-case only the torques and not the forces from $F_{\text {sen }}$ were measured, in order to analyse the performance of the force controller on torques The graphs in Figure 14 show the starting position and final position of the cup in this test.

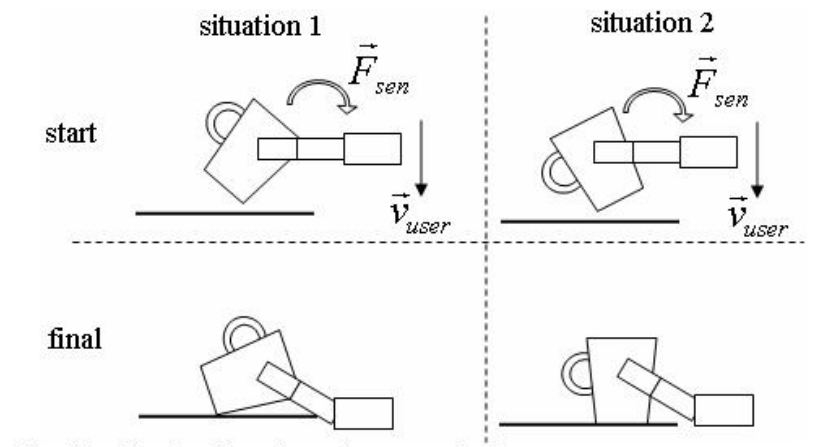

Fig. 14. Steering the gripper to open a turning cap

In situation 1 (left) the cup is rotated clockwise with respect to the table, in situation 2 (right) the cup is rotated anti-clockwise. Situation 2 of Figure 14 shows that the force controller helps the user in putting the cup on the table. In situation 1 , however the situation becomes more difficult for the user. Reason for this is that the measured torque in both situations is clockwise. This means that if the cup is rotated anti-clockwise, situation 2, force control helps. If the cup is already rotated clockwise, situation 1 , the cup will be rotated even more clockwise due to the force controller. It can therefore be concluded that force control using measured torques is not helping the user in all cases. Therefore it is not implemented in the simultaneous position/force controller of the ARM.

\section{ConClusions}

The aim was to design an assistant steering strategy based on force control to help the user operate the ARM. The result is a simultaneous position/force controller based on the humans control strategy of their hand. The controller is not applicable to the ARM only, but it can be implemented in any other (similar) robot. The innovative element is the single integration of the measured forces together with the virtual box in which force control is allowed. Assets of the designed control strategy are:

- The performance of the controller does not depend on the amount of backlash in the drive train of the robot,

- The controller can be used in both rigid robot as well as non-rigid ones.

The test-case 'pressing a button' showed that this simultaneous position/force controller reduces the impactforce and -time of the robotic manipulator. From the testcases 'opening a door' and 'opening a turning cap' can be concluded that the force controller assists the user with complex manipulations. From the test-case 'putting a cup on the table' became clear that only the forces and not the torques should be controlled. When also torques are followed by the force controller the performance depends on the task.

\section{REFERENCES}

[1] Catalogue of ATI Automated Instruments, Apex, NC, USA, 2006, pp. 20-21

[2] A. R. Konuk, "Development and Implementation of Compliant Controller On Mamus Robot Arm," M.Sc Thesis, Univ ersity of Twente, 2004

[3] K. Ohishi, M. Miyazaki and M. Fujita, "Hybrid Control of Force and Position without force sensor," Proc. of 1991 IEEE Int. Conf. on Automatic Control, Vol 2, pp. 1049-1054, 1991.

[4] S. B. Karunakar and A. A. Goldenberg, "Contact Stability in Madel Based Force Control Systems of Robot Manipulators," Proc. of 1988 IEEE Int. Symp. on Intelligent Control, pp. 412417, 24-26 Aug 1988

[5] B. Driessen, F. Liefhebber, T. T. Kate and K. Van Woerden, "Collabrative Control of the MANUS Manipulator", Proc. of 2005 IEEE Int Conf on Rehabilitation Robotics, pp. 247-251, 28 June-2 July 2005

[6] G. R. B. E. Römer, H. J. A Stuyt and A Peters. "Cost-Savings and Economic Benefits due to the Assistive Robotic Manipulator (ARM)" Proceedings of the 2005 IEEE 9th International Conference on Rehabilitation Robotics, June 28 - July 1, 2005, Chicago, IL, USA, pp. 201-204.

[7] G. Zeng and A. Hemami, "An overview of robot force control", Cambridge University Press, Robotica, Vol 15, pp. 473488, September 1997 